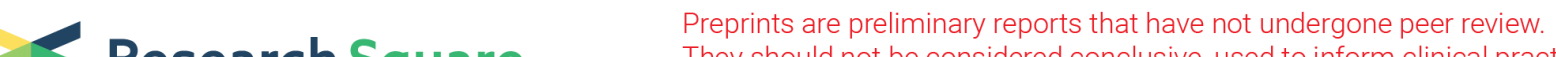 \\ They should not be considered conclusive, used to inform clinical practice, or referenced by the media as validated information.
}

\section{Anti-tumor effect of CTLA-4 antibody is independent of checkpoint blockade}

\section{Han Gao}

Beijing Normal University

\section{Haiyan Cai}

Shanghai Jiao Tong University School of Medicine

\section{Fei Zhang}

Shanghai Jiao Tong University School of Medicine

\section{Mingdong Liu}

Shanghai Jiao Tong University School of Medicine

\section{Xiaoxiao Wang}

Alphamab Co. Ltd.

\section{Pilin Wang}

Alphamab Co. Ltd.

\section{Fei Dou}

Beijing Normal University https://orcid.org/0000-0003-2003-6011

\section{Fubin Li}

Shanghai Jiao Tong University School of Medicine https://orcid.org/0000-0001-6268-3378

\section{Ting Xu}

Alphamab Co. Ltd.

Aiwu Zhou ( $\square$ awz20@shsmu.edu.cn )

Shanghai Jiao Tong University School of Medicine https://orcid.org/0000-0002-2555-5091

\section{Article}

Keywords: CTLA-4, antibodies, ipilimumab, immunotherapy

Posted Date: September 21st, 2021

DOI: https://doi.org/10.21203/rs.3.rs-907762/v1

License: (9) This work is licensed under a Creative Commons Attribution 4.0 International License. Read Full License 


\section{Abstract}

Antibodies targeting CTLA-4 are emerging as an important class of cancer therapeutics. It is assumed that these antibodies cause tumor rejection by blocking negative signaling from the CTLA-4-B7 interactions to enhance the priming of naïve $T$ cells in lymphoid organs. However, recent findings have shown that the effectiveness of CTLA-4 antibody critically depends on the Fc domain and the host Fc receptors. It remains unclear if the blocking function of CTLA-4 antibody is required for its anti-tumor activity. To address this, here we have selected a non-blocking anti-CTLA-4 antibody (D138) and assessed its binding property and antitumor activity in comparison with the therapeutic CTLA-4 antibody ipilimumab. Crystal structures of CTLA-4 complexed with these antibodies show that D138 binds to a distinctly different site to that of ipilimumab on the CTLA-4 surface. D138 binding did not block the association of cells expressing CTLA-4 and B7 whereas ipilimumab did. Subsequent antitumor assay revealed that D138 was similarly effective as ipilimumab in inhibiting tumor growth in mice. This antitumor activity required Fc function for efficacy and was correlated with selective reduction of intratumor regulatory $T\left(T_{\text {reg }}\right)$ cells, resulting in a significant increase in the ratio of $C D 8^{+}$over $T_{\text {reg }}$ cells. Overall these data clearly demonstrate that blocking CTLA-4-B7 interaction is not required for CTLA-4 antibody mediated antitumor activity, opening prospects of developing non-blocking CTLA-4 antibodies or simple binders towards other $\mathrm{T}_{\text {reg }}$ surface markers for $\mathrm{T}_{\text {reg }}$-targeted immunotherapy.

\section{Background}

Cytotoxic T-lymphocyte antigen-4 (CTLA-4), also known as CD152, is an immune-inhibitory molecule expressed in activated $\mathrm{T}$ cells and in suppressor $\mathrm{T}$ cells $\mathrm{s}^{1-3}$. It outcompetes the stimulatory receptor CD28 for binding to its ligands B7 (CD80 and CD86) with higher affinity ${ }^{4}$. Interaction of CTLA-4-B7 serves as a natural brake, thereby altering both T-cell proliferation and downstream cytokine production ${ }^{5}$. CTLA-4 ligation is also important for the immune suppressive function of regulatory $T\left(T_{\text {reg }}\right)$ cells, further assisting to dampen $T$ cell responses ${ }^{6}$. Therefore, it is assumed that blockage of the interaction between CTLA-4 and its ligands B7 should remove the CTLA-4 inhibitory signal and release a brake on the immune system, allowing a natural immune response to react to cancer cells ${ }^{7,8}$. A specific CTLA-4 monoclonal antibody $(\mathrm{mAb})$ developed on this assumption has emerged as an effective therapy for cancer treatment. Ipilimumab (Fig. 1a), a fully humanized antibody targeting CTLA-4, received FDA approval for the treatment of metastatic melanoma in 2011. Another anti-CTLA-4 mAb, tremelimumab is also in multiple phase III clinical trials ${ }^{9}$. The previous structural studies confirmed that the surfaces of CTLA-4 occupied by these two antibodies overlapped with that of B7 on the CTLA-4 indicating binding of these antibodies on CTLA-4 would block the interaction between CTLA-4 and B $7^{10-12}$.

However, recent findings have shown that the effectiveness of these CTLA-4 antibodies in mice critically depends on the Fc domain and the cell surface expressed Fc receptors ${ }^{13-16}$. The anti-tumor activity of 
these antibodies is associated with selective depletion of $T_{\text {reg }}$ cells within tumor lesions through $\mathrm{Fcy}$ receptor-expressing leukocytes ${ }^{14-16}$. Also, in patients with advanced melanoma, response to ipilimumab correlates with the $\mathrm{Fc}$ receptor high affinity polymorphism (CD16a-V158F) ${ }^{17}$. Furthermore, it was reported that a blocking nanobody $\mathrm{H} 11$ without $\mathrm{Fc}$ has very limited antitumor activity in mice ${ }^{18}$. Therefore, it remains unclear if the blocking function of CTLA-4 antibodies is required for their anti-tumor activity or if a non-blocking CTLA-4 antibody would be active in inhibiting tumor growth. To address this, we have solved the crystal structure of a non-blocking CTLA-4 nanobody (D138) complexed with CTLA-4 and the crystal structure ipilimumab complexed with CTLA-4, and compared their binding properties and antitumor activities. This demonstrates that D138, a non-blocker not interfering with the hemostasis of CTLA4 and its interactions with B7 on cell surface, is similarly effective as ipilimumab in inhibiting tumor growth in mice. These findings open opportunities in selecting non-blocking CTLA-4 antibodies for cancer immunotherapy.

\section{Results}

\section{Selection of a novel non-blocking CTLA-4 antibody}

In order to obtain high affinity CTLA-4 antibody, camels were immunized with human CTLA-4 and a phage library derived from peripheral blood mononuclear cells (PBMC) was generated and screened for anti-CTLA-4 nanobody or domain antibodies (dAb). One of these antibodies binds human CTLA-4 with a modest dissociation constant (Kd) of $25.3 \mathrm{nM}$ (Fig. 1a, 1b) when measured by Bio-layer interferometry (BLI) and shows little interference on the binding between CTLA-4 and B7 during screening. This nanobody fused with human IgG1 Fc as seen in ipilimumab, was termed D138 and selected for further characterization (Fig. 1a). In a competitive binding ELISA assay, CTLA-4 was coated on the plates and a mixture of B7-1 or B7-2 with different concentrations of antibody was then added into the wells. The binding of $B 7$ on the plates was subsequently quantified and plotted as seen in Fig. 1c. This showed ipilimumab blocked the binding of B7-1 with an $\mathrm{IC}_{50}$ of $24.6 \mathrm{nM}$ and of B7-2 with an $\mathrm{IC}_{50}$ of $10.4 \mathrm{nM}$, while D138 had little blocking effect.

We further compared the binding properties of these antibodies by BLI assay (Fig. 1d). CTLA-4 was anchored on a sensor and loaded with ipilimumab or D138 for 200 seconds (stage I) and then washed with buffer for 60 seconds (stage II). These two stages represented the typical antibody binding curves of association and dissociation (Fig. 1d). Subsequently the sensors with preformed CTLA-4-antibody complexes were dipped into the antibody solution again to allow further association or into the antibody solution of same concentrations but with added B7-1 to check if B7-1 would bind (stage III). As expected, there was slight increase in the signal from sensor when loaded again with corresponding antibodies indicating further antibody binding onto the sensors (Fig. 1d, black curves). However, in the presence of B7-1 there was a significant association signal from the sensor with preformed D138/CTLA-4 complexes (Fig. 1d, blue curve) indicating significant binding of B7-1 onto CTLA-4 in the sensor surface. This result 
demonstrates that B7-1 could readily bind to the preformed CTLA-4-D138 complex, and binding of D138 would not block CTLA-4 from binding B7-1. This is different to ipillimumab where little binding of B7-1 towards preformed CTLA-4-ipilimumab complex on the sensor was observed ${ }^{19}$. This suggests that ipilimumab is a blocker although there were some doubts on this ${ }^{20}$ and that D138 is a non-blocker.

\section{Crystal structure of CTLA-4 complexed with D138-dAb or ipilimumab}

To elucidate the structural mechanism underlying the binding interaction between CTLA-4 and D138, we prepared recombinant dimeric CTLA-4 complexed with D138-dAb, which could be separated from CTLA-4 monomer complexed with D138-dAb by ion-exchange chromatography, and solved its crystal structure at a resolution of $3.25 \AA$ (Fig. 2a, Supplementary Table 1). The structure showed a tetramer containing a CTLA-4 homo dimer and two molecules of D138-dAb (Fig. 2b). The overall structure of CTLA-4 dimer in the complex is similar to its apo form (PDB 30SK) ${ }^{21}$ with a RMSD of $2.2 \AA$ indicating D138 binding induced little conformational changes in CTLA-4. D138-dAb has a typical nanobody conformation with a long complementarity determining region (CDR3) loop of 19 residues (Supplementary Fig. 1a, b). It binds to a concave surface area formed by the connecting loops of the front and back beta-sheets of the CTLA4 molecule with a total buried surface area of $760 \AA^{2}$ (Fig. 2c, d, e). The binding interactions involve residues from all three CDR loops of D138. Notably, Val54 of CDR2 loop in D138 forms key hydrophobic interactions with the shallow pocket in CTLA-4 involving sidechains of Thr47, Val49, lle67 and Tyr92 (Fig. 2d). Replacement of Thr47 and lle67 with Ala in CTLA-4 resulted 14 and 26-fold decrease in binding affinity respectively, while replacement of Val49 and Tyr92 with Ala resulted in a complete loss of the binding affinity towards D138. There are also eleven hydrogen bonds or salt bridges formed between D138-dAb and CTLA-4 (Supplementary Table 2). Glu48 on the tip of C' strand of CTLA-4 forms a hydrogen bond with the indole ring nitrogen atom of Trp105 in the CDR3 of D138-dAb (Fig. 2c, Supplementary Fig. 1C). Asp64 and Asp65 in C"D loop of CTLA-4 form salt bridges with Arg47 and Arg108 in CDR3 (Fig. 2C). Also Asp88 of DE loop in CTLA-4 formed a hydrogen bond with the main chain nitrogen atom of Gly55 in D138-dAb CDR2 region. Replacement of these three residues with alanine respectively dramatically decreased or abolished the binding between D138 and CTLA-4 (Fig. 2d, Supplementary Table 3).

As the binding site of B7 on CTLA-4 is centered around the FG loop near the lower part of the front betasheet (Fig. 2e), there is no overlap between the binding surfaces of B7 and D138. Superimposition of the structures of their complexes on CTLA-4 also showed that there would be little steric clash between D138 and B7 within the CTLA-4-B7-D138 ternary complex (Fig. 2f, Supplementary Fig. 1d). This is consistent with our observation above that D138-dAb is a non-blocker and it can bind to preformed CTLA-4-B7 complex (Fig. 1d). 
The crystal structure of ipilimumab Fab or ScFv domain complexed with CTLA-4 has been solved previously ${ }^{10,11}$, however there were some concerns if ipilimumab is really a blocker of CTLA-4-B7 interaction ${ }^{20}$. Here we crystallized ipilimumab-Fab/CTLA-4 complex under a different condition with a different space group and solved its structure at higher resolution (2.5 $)$. There were two copies of ipilimumab Fab fragment/CTLA-4 complexes within the asymmetric unit (Supplementary Fig. 1e). The overall structure of the complex resembles the previously published structures with a buried CTLA-4 surface area of $870 \AA^{2}$ within the complex regardless the differences in crystallization conditions and resolution of the structures ${ }^{10,11}$. The ipilimumab binding site on CTLA-4 is located close to the lower part of the front beta-sheet and overlaps with that of B7 binding site (Fig. 2e). When superposed together, there is a clear steric clash between ipilimumab and B7 (Fig. 2f). Therefore, ipilimumab is undoubtedly a blocker of CTLA-4-B7 interactions.

\section{D138 does not interfere with CTLA-4-B7-mediated cell-cell interactions}

To further evaluate the effect of D138 and ipilimumab on the interactions of CTLA-4 and B7 on cell surface, cells expressing a human CTLA-4 mutant fused with RFP, where the CTLA-4 cytoplasmic tail of 35 amino acid was replaced by that of PD-L1 to abrogate the spontaneous endocytosis of CTLA-4 and thus allow stable cell surface expression ${ }^{22}$, were mixed with cells expressing B7-1-GFP in the absence or in the presence of the antibodies. The cell-cell association was then followed by fluorescent microscope and flow cytometry with cells expressing CTLA-4-RFP showing red fluorescence while cells expressing B71 fused with GFP showing green fluorescence (Fig. 3a). When the cells were mixed at a ratio of 1:1 directly, they readily aggregated to form clumps upon mixing due to binding interactions between cell surface expressed CTLA-4 and B7-1 as examined by fluorescent microscope. Pretreatment of CTLA-4-RFP expressing cells with $100 \mathrm{nM}$ ipilimumab completely blocked the aggregation formation (Fig. 3b). In contrast, pretreatment with D138 or control human IgG had no effect on the cell aggregation. This confirms that ipilimumab is a blocker of CTLA-4-B7 interactions on cell surfaces while D138 is not, which is consistent with a model of their complex on the cell surface (Supplementary Fig. 1d).

The dose-dependent blocking effect of ipilimumab and D138 was further assessed by flow cytometry analysis. Cells expressing CTLA-4-RFP ( $2^{\prime} 10^{5}$ cells) were pretreated with different concentrations (0-200 $\mathrm{nM})$ of antibodies for $5 \mathrm{~min}$, then mixed with cells expressing B7-1-GFP $\left(1^{\prime} 10^{6}\right.$ cells $)$ and subjected to flow cytometry analysis for the tethered cells with both GFP and RFP signals. The percentage of the GFP RFP cells over total RFP ${ }^{+}$cells was plotted against the concentrations of the antibodies. As shown in Fig. 3c, when cells expressing B7-1-GFP were mixed with cells expressing CTLA-4-RFP, about 80\% of CTLA-4 expressing cells became tethered with B7-1 expressing cells and showed strong signal for both GFP and RFP. Addition of $100 \mathrm{nM}$ ipilimumab largely blocked the formation of tethered cells while no effect was observed with the addition of D138 or control IgG. The IC ${ }_{50}$ value of ipilimumab mediated blockade of 
cell-cell interactions was $13.8 \mathrm{nM}$ (Fig. $3 \mathrm{~d}$ ) as calculated from the dose-dependent curve, which is largely consistent with $\mathrm{IC}_{50}$ value derived from the ELISA assay (Fig. 1C). Altogether these data further confirm that D138 is a simple CTLA-4 binder and that it does not block CTLA-4-B7 binding on cell surface.

Since wild type CTLA-4 when expressed on the cell surface readily recycles between surface and endosomes ${ }^{22,23}$, we tested if ipilimumab or D138 binding would perturb this process. Cells stably expressing human CTLA-4 were treated with different concentrations of antibodies respectively and then checked for the amount of surface expressed CTLA-4 by flow cytometry. Interestingly we found that ipilimumab binding reduced the surface expressed CTLA-4 while D138 had no effect (Fig. 3e). This clearly demonstrates that D138 binding does not perturb the normal hemostasis of CTLA-4 on cell surface while ipilimumab does. As the binding of both antibodies towards CTLA-4 is not pH sensitive (data not shown) with their complexes unlikely affected by $\mathrm{pH}$ changes during endocytosis process, the different effect of D138 and ipilimumab on cell surface CTLA-4 likely arises from the difference in the binding epitopes of these two antibodies on CTLA-4.

\section{Biological activity of D138}

It is known that binding of CTLA-4 to the costimulatory molecules inhibits T-cell activation by reducing interleukin-2 (IL-2) production and IL-2 receptor expression, and by arresting T cells at the G1 phase of the cell cycle, thus an immune checkpoint blocker such as ipilimumab or tremelimumab would block CTLA-4B7 inhibitory pathway and enhance IL-2 production ${ }^{9,24}$. Here we tested if D138 would have a similar effect. PMBCs from healthy donors were mixed with ipilimumab or D138 of different concentrations for one hour and then stimulated with Staphylococcal Enterotoxin B (SEB) super antigen. The concentrations of the cytokines such as IL-2, IFN- $y$ and TNF- $a$ in the supernatant were measured after 5 days of culture. As expected, ipilimumab induced secretion of IL-2 dose-dependently with significant IL-2 production detected at the concentration of $200 \mathrm{nM}$. However, there was very limited secretion of IL-2 from D138 treated samples (Fig. 4a). There were no significant secretions of IFN-y or TNF-a when treated with either ipilimumab or D138 consistent with previous studies of ipilimumab (Supplementary Fig. 2a and 2b).

As both D138 and ipilimumab could bind CTLA-4 and have the same Fc domain, we then assessed their antibody-dependent cellular cytotoxicity (ADCC) effects on the viability of co-cultured $T_{\text {reg }}$ cells from PBMC. This showed that both antibodies could induce $T_{\text {reg }}$ cell lysis leading to increased lactate dehydrogenase (LDH) release (Fig. $4 b$ ). It appeared that ipilimumab has stronger ADCC activity than D138 (Fig. 4b). When the ADCC activities of these antibodies were evaluated on engineered effector cells carrying a reporter gene with binding sites for the principal transcription factors involved in FcyRIIIA signal transduction, D138 and ipilimumab showed similar activity (Supplementary Fig. 2c). Overall, these 
results showed ipilimumab could induce IL2 release from immune cells and induced ADCC activity while D138 could not acviated IL-2 release from immune cells, but could induce similar ADCC activity as ipilimumab.

\section{Antitumor effect of D138 and ipilimumab is associated with $T_{\text {reg }}$ depletion within tumor lesions}

Subsequently, the in vivo antitumor activities of D138 and ipilimumab were assessed against MC38 colon adenocarcinoma tumor in human CTLA-4 knock-in mouse model, with treatment initiated 9 days after tumor implantation. Tumor volumes and diameters were recorded every three days. At a relatively low dose of $0.8 \mathrm{mg} / \mathrm{kg}$, ipilimumab showed a significant tumor inhibition activity when compared with the control group treated with human IgG (Fig. 5a, b). Most remarkably, similar therapeutic effects of D138 was also observed with D138 treatment $(0.5 \mathrm{mg} / \mathrm{kg}$, same numbers of molecules as ipilimumab) (Fig. 5a, b). However, the Fc mutants of both antibodies (ipilimumab-AG or D138-AG), where double mutations (D265A, P331G) in Fc (FcAG) largely abolished the binding of Fc receptors ${ }^{25}$, showed little antitumor activities. This indicates that the blocking function is not required for the anti-tumor activity of CTLA-4 antibody, while the Fc receptor binding ability is critical for efficient tumor inhibition.

It has been well documented that the anti-tumor activities of CTLA-4 antibodies in mice is often associated with depletion of $T_{\text {reg }}$ cells within the tumor micro environment ${ }^{14-16}$. Here we tested if a similar effect could be observed with D138. Although $\mathrm{T}_{\text {reg }}$ depletion could occur within days after antibody injection, in order to correlate the tumor growth inhibition with the level of $T_{\text {reg }}$ within tumor lesions in each mouse, mice ( $\mathrm{n}=10$ each group) with implanted tumors were treated with four injections of antibodies or control IgG and tumor growth was followed (Supplementary Fig. 3a). Whereas tumors grew progressively in the control IgG-treated mice and very limited growth inhibition was observed in the D138AG and ipilimumab-AG groups, significant tumor growth retardation was seen with both D138 and ipilimumab-treated groups. The tumors retrieved from these two groups were smaller than those of other groups (Supplementary Fig. 3b). These results are consistent with the anti-tumor effect seen in Fig. 5a and Fig. $5 b$.

The tumors were then digested and $\mathrm{T}$ cell populations were analyzed by flow cytometry using various $\mathrm{T}$ cells markers (Fig. 5c-5i and Supplementary Fig. 3). We firstly confirmed that high level of CTLA-4 expression is associated with Foxp $3^{+}$but not Foxp3 ${ }^{-}$cells in the $\mathrm{CD} 4^{+}$subset (Supplementary Fig. $3 \mathrm{C}$ ). There were no changes in the $\mathrm{CD} 4^{+}$Foxp3 ${ }^{-}$cell population amongst all the treated groups (Supplementary Fig. 3e), but there was a significant decrease in the $C D 4^{+} \mathrm{Foxp}^{+}$subpopulation (termed $\mathrm{T}_{\text {reg }}$ cells here) in tumors treated by ipilimumab or D138 as reflected in the absolute numbers of these cells (Fig. $5 \mathrm{~d}$ ) or the percentage of $\mathrm{T}_{\text {reg }} / \mathrm{T}$ cells (Supplementary Fig. $3 \mathrm{~d}$ ) or $\mathrm{T}_{\text {reg }} / \mathrm{CD} 4^{+}$cells (Fig. 5e). This was consistent with the decreased MFI of OX40 on $T_{\text {reg }}$ cell surface in these two groups (Supplementary Fig. $3 \mathrm{~g}$ ). In contrast, 
there was a slight increase in the total T cell numbers (Supplementary Fig. $3 f), \mathrm{CD}^{+}$cell numbers (Fig. 5f) and in the percentage of $\mathrm{CD}^{+} / \mathrm{T}$ cells (Fig. $5 \mathrm{~g}$ ) in the tumors treated with ipilimumab and D138 antibodies. This cumulatively led to a near 10 -fold increase in the ratio of $\mathrm{CD}^{+}$over $\mathrm{T}_{\text {reg }}$ cells (Fig. $5 \mathrm{~h}$ ). To further correlate the changes in the ratio of $\mathrm{CD}^{+} / \mathrm{T}_{\text {reg }}$ with tumor growth in each tumor, the ratios were plotted against the changes in size as shown in (Fig. $5 \mathrm{i}$ ). This clearly demonstrated little changes in the ratio of $\mathrm{CD} 8^{+} / \mathrm{T}_{\text {reg }}$ cells from the control IgG or the D138-AG treated tumors, but significant changes in the ipilimumab and D138 treated tumors. Interestingly, there was also a small decrease in total $T_{\text {reg }}$ cell numbers in ipilimumab-AG treated tumors (Fig. 5c) with some of these tumors having slightly increased $\mathrm{CD}^{+} / \mathrm{T}_{\text {reg }}$ ratios (Fig. $5 \mathrm{i}$ ), which might reflect certain role of the blocking function of ipilimumab-AG. Nevertheless, these results show that antitumor effect of D138 and ipilimumab is associated with depletion of $T_{\text {reg }}$ cells within the tumor microenvironment and this selective depletion mainly depends on the function of Fc.

\section{Discussion}

It is generally accepted that anti-CTLA-4 monoclonal antibodies induce tumor rejection by blocking negative signaling from the CTLA-4-B7 interaction 7,8 , thus these antibodies are often called immunecheckpoint blockers. Most, if not all, of these antibodies in clinical trials are selected based on their blocking abilities and the Fc mediated functions were not considered to be critical to their antitumor activities $^{26}$. However, many recent studies have shown that the anti-tumor activities of these antibodies, including ipilimumab, critically depend on the presence of Fc domain and Fc receptors on the cell surface $^{13-15,17,18}$. It was also suggested by Du et al that ipilimumab functioned through depletion intratumor $\mathrm{T}_{\text {reg }}$ instead of blocking CTLA-4-B7 pathway in mouse, but surprisingly they found that ipilimumab failed to block CTLA-4-B7 interactions ${ }^{20}$. Nevertheless, it appeared that the blocking function of CTLA-4 antibodies played a minor role in the anti-tumor activity. To dissect the blocking and Fc mediated functions of CTLA-4 antibodies, here we selected a non-blocking CTLA-4 antibody D138 (Fig. 1) and compared its binding ability and antitumor activities with those of ipilimumab. Our crystallographic and in vitro binding assays unequivocally demonstrated that D138 is a simple binder of CTLA-4 and does not impede CTLA-4-B7 interactions on cell surface while ipilimumab is a checkpoint blocker (Fig. 3).

Remarkably, in vivo assay in human CTLA-4 knock-in mouse model showed both antibodies had similar antitumor activity which were associated with a selective depletion of intratumor $\mathrm{T}_{\text {reg }}$ cells and a significant increase in the ratio of $\mathrm{CD}^{+} / T_{\text {reg }}$ cells within the tumor lesions. Furthermore, both antibodies required intact $\mathrm{Fc}$ for tumor inhibition activity as their mutants with impaired Fc receptor binding lost antitumor activity (Fig 5a, Supplementary Fig 3a,b). Thus, the main contributing factor of the antitumor activity from both antibodies is the $\mathrm{Fc}$ mediated $\mathrm{T}_{\text {reg }}$ depletion with blocking function of ipilimumab not essential, which is consistent with previous observations ${ }^{18,20}$. Although this selective intratumor $\mathrm{T}_{\text {reg }}$ 
depletion has been shown in various mouse models, it remains less clear if CTLA-4 antibodies such as ipilimumab function through this route in human mainly due to the difficulties in obtaining large numbers of tumor samples from patients before and after treatment ${ }^{17,27,28}$. Nevertheless, directly targeting intratumor $\mathrm{T}_{\text {reg }}$ cells represents an attractive approach for clinical cancer treatment ${ }^{29}$. A transcriptome analysis of human cancer specimens has revealed that tumor infiltrating $T_{\text {reg }}$ cells were highly suppressive and expressed other specific signature molecules such as OX40, TIGIT, PD-1, GITR and CCR8 etc $^{30}$. Simple binders, instead of agonists or antagonists or blockers, targeting those intratumor $\mathrm{T}_{\text {reg }}$ surface markers would fit this purpose in inducing intratumor $\mathrm{T}_{\text {reg }}$ depletion as well as CTLA-4 blocking antibodies.

However, D138 may hold some advantages over CTLA-4 blockers such as ipilimumab for cancer treatment. CTLA-4 is constitutively expressed in $T_{\text {reg }}$ cells, but at any given time only about $10 \%$ of total CTLA-4 is present on the cell surface with most of CTLA-4 located in the endocytosis recycle pathway within the cell ${ }^{31}$. It has been reported that binding of blocking antibodies on CTLA-4 on the cell surface induces CTLA-4 internalization and the detailed molecular mechanism underlying this process is not well understood ${ }^{23,32}$. Here we confirmed that the cell surface binding of ipilimumab induced CTLA-4 internalization with a reduction of CTLA-4 molecules on cell surface, however, to our surprise, binding of D138 on the cell surface did not induce similar reduction of CTLA-4 (Fig. 3e). As normal function of CTLA-4 in peripheral tissues is critical for maintaining the homeostasis of the immune system, deficiency in CTLA-4 expression in human is often associated with autoimmune diseases ${ }^{33,34}$. Also clinical use of ipilimumab is closely related to immunotherapy-related adverse events (irAE) ${ }^{35-37}$ and it was recently proposed that these side effects could arise from the disturbance in the recycling of CTLA-4 in the cell ${ }^{38}$. Since D138, a simple binder identified here with strong anti-tumor activity, would neither perturb the homeostasis of CTLA-4 on cell surface, nor block the normal interactions between CTLA-4 and B7, it may induce less side effects than the CTLA-4 blocking antibodies. Therefore, the simpler could be the better, and this opens the prospects of developing simple binders towards $T_{\text {reg }}$ surface markers for $T_{\text {reg }}$-targeted immunotherapy.

\section{Declarations}

\section{Acknowledgements}

We thank the staff at the SSRF BL17U1 and BL19U1 for their assistance during data collection. We thank professors Robin W Carrell and Randy J Read for reading through the manuscript.

\section{Conflicts of Interest}

X.W., P.W. and T.X. are employees of Alphamab Co. Ltd. 


\section{Funding}

This work was supported in part by grants from the National Basic Research Program of China (973 Program, 2015CB554203), the National Natural Science Foundation of China (81502981, 81572090 and 32070934) and Science Foundation for The Excellent Youth Scholars of Shanghai (zzjdyx14007).

\section{Author Contributions}

H.G, H.C. F.Z. prepared all related proteins and solved the crystal structures of CTLA-4 complexed with antibodies.

H.G., M.L. and F.L. performed in vivo study on mouse model and flow cytometry analysis of intratumor T cell populations.

X.W. P.W. and F.D. screened nanobody library, identified and characterized D138.

A.Z. and T.X. supervised the study and wrote the manuscript along with input from F.D. and F.L.

\section{Materials And Methods}

\section{Cell lines and culture}

The MC38, HEK293T and CHO cell lines were all purchased from American Type Culture Collection (ATCC). MC38 and HEK293T cell lines were cultured in Dulbecco's Modified Eagle Medium (DMEM, Gibco) supplemented with $10 \%$ Fetal Bovine Serum (FBS, Gibco) and $100 \mathrm{mg} / \mathrm{ml}$ penicillin (Sigma) and $100 \mathrm{mg} / \mathrm{ml}$ streptomycin (Sigma). CHO cell line was cultured in CD CHO Medium (Invitrogen). For protein expression, HEK293T was culture in suspension in serum free medium (Gibco). All cell lines were kept at $37^{\circ} \mathrm{C}$ in a $5 \% \mathrm{CO} 2$ incubator.

C57BL/6J Ctla-4h/h female mice (6-8 weeks old) were purchased from Shanghai Model Organisms Center and maintained in a special pathogen-free environment and in individual ventilation cages. It is considered that there are no known contaminants in the dietary materials that could influence the tumor growth. The protocol and any amendment(s) or procedures involving the care and use of animals in this study were reviewed and approved by the Institutional Animal Care and Use Committee (IACUC) of Shanghai Model Organisms Center prior to conduct. During the study, the care and use of animals were conducted in accordance with the regulations of the Association for Assessment and Accreditation of Laboratory Animal Care (AAALAC). 


\section{Generation of camel nanobodies against CTLA-4}

Anti-CTLA-4 nanobodies were generated and prepared using similar procedure as previously described ${ }^{39}$. Camels (Camelus bactrianus) were immunized with injections of human CTLA-4 Fc fusion protein. Peripheral blood lymphocytes $(100 \mathrm{ml})$ were isolated one week after the last immunization. The DNA sequences corresponding to the variable domains of the heavy-chain antibodies were amplified with specific primers and used to create a nanobody phage display library. Enrichment screening against human CTLA-4 was performed in $96-$ well plates coated with $10 \mu \mathrm{g}$ of protein per well. High-affinity bacteriophages were obtained after 4 rounds of screening. Ninety-six individual colonies were randomly selected and amplified in culture with positive colonies sequenced. The interested nanobodies such as D138-dAb were fused with human IgG1 Fc and expressed in HEK293T cells. Ipilimumab variants, CTLA-4 fused with mouse Fc or human Fc and B7-fusion proteins were similarly expressed in HEK293T cells and purified by a protein $\mathrm{G}$ column.

\section{Binding affinity measurement of D138 with CTLA-4 variants}

The measurement was performed on Octet K2 through Bio-layer interferometry (BLI). The recombinant anti-human CTLA-4 antibody D138 was diluted to $10 \mathrm{mg} / \mathrm{ml}$ and anchored on the Anti-Human Fc Capture (AHC) biosensor for 120 seconds with a threshold $2 \mathrm{~nm}$ thickness. Human CTLA4-mFc or CTLA-4-His variants was then serially diluted ( $200 \mathrm{nM}, 100 \mathrm{nM}, 50 \mathrm{nM}, 25 \mathrm{nM}, 12.5 \mathrm{nM})$, loaded for 120 seconds, dissociated for 300 seconds and regenerated for 5 seconds with $10 \mathrm{mM}$ glycine- $\mathrm{HCl}(\mathrm{pH} \mathrm{1.7)}$. Binding rate $\left(\mathrm{K}_{\mathrm{on}}\right)$ and dissociation rate $\left(\mathrm{K}_{\mathrm{off}}\right)$ were calculated using a simple one-to-one Languir binding model (Octet K2 Data Analysis Software Version 9.0). The equilibrium dissociation constant (Kd) was calculated as the ratio $\mathrm{K}_{\text {off }} / \mathrm{K}_{\text {on }}$.

\section{Evaluation of the effect of antibodies on CTLA-4 and B7 binding}

For the ELISA assay to evaluate if CTLA-4 antibodies would block the interaction between CTLA-4 and B7, plates were coated with CTLA-4-human Fc fusion protein at $3 \mu \mathrm{g} / \mathrm{ml}$ with $3 \%$ BSA in PBS for 2 hours at room temperature. Mixtures containing antibodies (D138 or ipilimumab) of different concentrations and 50ng/ml B7-1- or B7-2-mouse Fc fusion protein in PBS plus 0.05\% Tween-20 and 1\% BSA were applied to the plate and incubated for additional 2 hours at $37^{\circ} \mathrm{C}$. The bound B7-1 or B7-2 was detected with the horseradish peroxidase (HRP)-conjugated goat anti-mouse lgG1, which was subsequently measured using tetramethylbenzidine (TMB) substrate with absorbance at $450 \mathrm{~nm}$. The mean value of triplicate wells ( \pm SD) was then plotted against the concentration of antibodies with $I C_{50}$ fitted (Fig. 1c). 
A BLI assay was also developed to assess the effect of antibody on CTLA-4-B7 binding. Human CTLA-4 fused mouse Fc (CTLA-4-mFc) protein was diluted to $10 \mathrm{mg} / \mathrm{ml}$, anchored on the Anti-Mouse Fc Capture (AMC) biosensors for 150 seconds, loaded with ipilimumab (50 nM) or D138 (50 nM) for 200 seconds (stage I) and then washed with PBST for 60 seconds (stage II). Subsequently the biosensors were dipped into the same antibody solution or a antibody solution of same concentration with added $200 \mathrm{nM} \mathrm{B7-1-FC}$ for 200 seconds (stage III). The biding response sensorgram were followed (Fig. 1d).

\section{Crystallization and structural study of CTLA-4 complexed with antibodies}

The Fab fragment of ipilimumab was produced in HEK293T mammalian expression system ${ }^{40}$. The cDNA encoding the heavy chain $(\mathrm{VH}-\mathrm{CH} 1)$ with $8 \times \mathrm{His}$ tag and light chain $(\mathrm{VL}-\mathrm{CL})$ of ipilimumab were cloned in pCEP4 expression vector respectively. They were co-transfected into HEK293T suspension cells using Polyethylenimine (PEI). The secreted Fab fragment in the medium was purified by a Ni-NTA column (Roche) following standard protocol.

Recombinant non-glycosylated CTLA-4 (amino acids 3-121) was prepared from E. coli using expression vector pET28a following refolding. Briefly, E.coli BL21 (DE3) cells harboring pET28a-hCTLA-4 expression construct were cultured at $37^{\circ} \mathrm{C}$ in LB and induced with $0.5 \mathrm{mM}$ isopropyl- $\beta$-D-thiogalactoside (IPTG) once the optical density at $600 \mathrm{~nm}$ reached 1.0 . After a further 16 -hour incubation at $37^{\circ} \mathrm{C}$, the cells were collected by centrifugation, resuspended in lysis buffer $(20 \mathrm{mM}$ Tris- $\mathrm{HCl}, \mathrm{pH} \mathrm{7.4,1 \%}$ Triton X-100, and 20 mM EDTA) and disrupted by sonication. Inclusion bodies were recovered by centrifugation at $15,000 \mathrm{~g}$ for 10 minutes and were then washed 3 times with lysis buffer, followed by washing with buffer without Triton X-100. The inclusion bodies were dissolved in $6 \mathrm{M}$ guanidine $\mathrm{HCl}, 0.5 \mathrm{mM}$ EDTA, and $10 \mathrm{mM}$ DTT, $20 \mathrm{mM}$ Tris- $\mathrm{HCl}, \mathrm{pH} 7.4$ and added drop-wise into a refolding buffer consisting of $1 \mathrm{M}$ Arg hydrochloride, $0.1 \mathrm{M}$ Tris $-\mathrm{HCl}, \mathrm{pH} 8.0,2 \mathrm{mM}$ EDTA, $0.25 \mathrm{mM}$ oxidized glutathione, $0.25 \mathrm{mM}$ reduced glutathione and $0.1 \mathrm{mg} / \mathrm{ml}$ of ipilimumab Fab fragment. The ipilimumab Fab/CTLA-4 complex was subsequently purified using ion exchange and gel filtration columns (GE Healthcare). The purified complex was concentrated to $\sim 15 \mathrm{mg} / \mathrm{ml}$ and the initial crystallization was performed with commercially available screening solutions through sitting-drop vapor diffusion method. After optimizing the initial crystallization conditions, crystals suitable for data collection were obtained at room temperature from $20 \%$ PEG $3000,0.2 \mathrm{M} \mathrm{Ca}(\mathrm{Ac})_{2}$ and 0.1M Hepes, pH 7.0.

Recombinant glycosylated human CTLA-4 ectodomain (amino acids 3-125) with a C-terminal His-tag and recombinant D138-dAb were co-expressed in HEK293T cells using a similar procedure as ipilimumab. The 
secreted protein in the medium was first purified by a Ni-NTA column and the eluted samples were then further purified by a SP ion exchange column (Fig. 2a). This separated CTLA-4 dimer complexed with D138-dAb from its monomer-D138-dAb complex. Purified CTLA-4 dimer complex was concentrated to $\sim 20 \mathrm{mg} / \mathrm{ml}$ and the initial crystallization was screened similarly as above and crystals suitable for data collection were obtained at room temperature from $12 \%$ PEG 3350, 3\% tascimate, pH 5.0.

Crystals were cryo-protected with $20 \%$ glycerol in the mother liquor and flash-cooled in liquid nitrogen. Diffraction data were indexed and processed with iMosflm and scaled with Aimless from the CCP4 suite $^{41}$. The initial phases were obtained by molecular replacement using Phaser ${ }^{42}$ with CTLA-4 (PDB 30SK), nanobody model (PDB 5JDS) and ipilimumab Fab (PDB 5TRU), respectively. The models were subsequently manually rebuilt using $\operatorname{Coot}^{43}$ and refine to good geometry using Refmac ${ }^{44}$. The atomic coordinates and structure factors have been deposited in the Protein Data Bank (PDB) (Supplementary Table S1). The binding interface between antibody and CTLA-4 was analyzed by PISA ${ }^{45}$. Figures were produced with PyMOL software ${ }^{46}$.

\section{Assessment the effects of antibodies on CTLA-4-B7 interaction on cell surface}

Human CTLA-4 fused with RFP, where the CTLA-4 cytoplasmic domain of 35 amino acids was replaced by that of PD-L1, and B7-1 fused GFP were transiently expressed on HEK293T cells, respectively. Cells expressing CTLA-4-RFP were treated with different concentrations of antibodies or control human IgG before mixing with cells expressing B7-1-GFP cells on ice for 5 minutes at an approximate ratio of 1:1 for fluorescence microscope analysis (Fig. 3b) or at 1:5 for flow cytometry analysis (Fig. 3c). In the flow cytometry analysis, the tethered cells with both GFP and RFP signal were counted and calculated against cells with RFP signal (Fig. 3d). The data was imported into Prism 7 (GraphPad) and analyzed using a four-parameter logistic (4PL) nonlinear regression curve.

\section{The effects of anti-CTLA-4 antibodies on the turn-over of CTLA- 4 on cell surface}

HEK293T cells stably expressing CTLA-4 were cultured in a 24 -well plate $\left(1.2^{\prime} 10^{5}\right.$ cells/well $)$ and antibodies were added at a concentration of $40 \mathrm{nM}$. After incubation for 1 or 6 hours at $37^{\circ} \mathrm{C}$, the supernatant was discarded and the cells were washed twice with PBS. A PE-labelled CTLA-4 antibody (diluted 1:50, Cat\# 349905, Biolegend), which did not affect the binding of either D138 or ipilimumab on CTLA-4, was then added. Thereafter, the cells were incubated at $4^{\circ} \mathrm{C}$ for $45 \mathrm{~min}$, and washed twice by PBS with $1 \%$ BSA. The cells were resuspended in $200 \mu \mathrm{l}$ of the same buffer and the PE fluorescence signal on the cell surface was measured by flow cytometry (Fig. 3e). All experiments were performed in triplicate wells for each condition and representative data are shown. 


\section{Analysis of IL-2, INF-y and TNF-a production}

PBMCs were obtained by Ficoll-Hypaque density gradient centrifuge from heparinized peripheral blood samples of the healthy donors. Freshly prepared PBMCs were plated in serum free RPMI-1640, and incubated in $37^{\circ} \mathrm{C}, 5 \% \mathrm{CO}_{2}$ for 2 hours in 96 -well plate $\left(2 \times 10^{5}\right.$ cells). The cells were then treated with different concentrations of ipilimumab or D138 for one hour. Staphylococcus Enterotoxin B (SEB, 200 $\mathrm{ng} / \mathrm{ml}$ ) was subsequently added. The supernatant was collected after five days culture and the levels of IL-2, INF- $y$ and TNF-a were evaluated by ELISA kit according to the manufacturer instructions.

\section{ADCC activities of Ipilimumab and D138}

We evaluated the ADCC activities of Ipilimumab and D138 using two methods, PBMC ADCC assays and ADCC Reporter Bioassay. PBMCs were obtained by Ficoll-Hypaque density gradient centrifuge from heparinized peripheral blood samples of the healthy donors. Freshly prepared PBMCs were plated in serum free RPMI- 1640 , and incubated in $37^{\circ} \mathrm{C}, 5 \% \mathrm{CO}_{2}$ for 2 hours in 96 -well plate $\left(2 \times 10^{5}\right.$ cells). $\mathrm{T}_{\text {reg }}$ cells were then separated from the PBMCs and incubated with CD3/CD28 and $10 \mathrm{ng} / \mathrm{ml} \mathrm{rhlL-2}$ for 8 days, which was used as target cells $\left(4 \times 10^{5}\right.$ cells $\left./ \mathrm{ml}\right)$. PBMCs obtained from another donor were used as effector cells. The PBMC were cultured and incubated with $30 \mathrm{ng} / \mathrm{ml} \mathrm{IL-2}$ for $22 \mathrm{~h}$, and then co-culture with Tregs cell at an effector-to-target ratio of 15:1, and along with different doses of CTLA-4 antibodies or control antibody. The effect of ADCC was measured by $L D H$ release after $4 \mathrm{~h}$ from treatments. The ADCC activities of antibodies were also determined using Fc Effector Reporter Bioassays. Jurkat cells with engineered expressions of FcyRIIIA (V158) and an NFAT (nuclear factor of activated T cells)-dependent firefly luciferase reporter gene that purchased from Promega (Madison, WI, USA) were used as effector cells. CTLA-4 transiently expressed on HEK293T cells were used as targeted cells. The effector cells ( $4 \times$ $10^{6} \mathrm{cell} / \mathrm{s} / \mathrm{ml}$ ) were co-cultured with target 293-CTLA-4 cells at an effector-to-target ratio of 4:1, and along with different concentrations of anti-human CTLA-4 antibodies or control. After incubation of the 96-well plates at $37^{\circ} \mathrm{C}$ for $6 \mathrm{~h}, 75 \mu \mathrm{l}$ Bio-Glo ${ }^{\mathrm{TM}}$ luciferase assay reagent (Promega) was added into each well and the luminescence intensity was measured with a plate reader (SpectraMax M3, Molecular Devices, CA, USA).

\section{Tumor growth and regression assay}

Age-matched C57BL/6J female mice with homozygous knock-in of the human CTLA-4 gene, were inoculated with colorectal cancer cell MC38 $\left(1^{\prime} 10^{6}\right.$ cells). After tumor size reached about $80 \sim 160 \mathrm{~mm}^{3}$, mice were grouped $(n=6)$ and treated four times (on day 9, 12, 15, 18 after inoculation of tumor cells) via intraperitoneal (i.p.) administration of anti-CTLA-4 antibodies $(0.8 \mathrm{mg} / \mathrm{kg}$ dose for ipilimumab or its FCAG 
mutant, $0.5 \mathrm{mg} / \mathrm{kg}$ for D138 or its FcAG mutant, $0.8 \mathrm{mg} / \mathrm{kg}$ for hlgG). The tumor growth and regression were determined by tumor volume as the readouts. Mice were humanely killed before the tumor volume reached $2500 \mathrm{~mm}^{3}$. The volumes $(V)$ were calculated using the following formula: $V=0.5^{\prime} a^{\prime} b^{2}$, where $a$ is the long diameter, while $b$ is the short diameter.

\section{Flow Cytometry analysis of T cell populations}

For analysis of $\mathrm{T}$ cell population within each tumor, $\mathrm{C} 57 \mathrm{BL} / 6 \mathrm{~J}$ mice were inoculated with MC38 cells as above and treated with four injections antibodies. The tumor-bearing mice $(n=10)$ were killed one day after the fourth immunotherapy (Supplementary Fig 2a). Tumor samples, spleen and lymph nodes were extracted from the mice. Each tumor was cut into small pieces, digested with Collagenase IV $(0.5 \mathrm{mg} / \mathrm{ml}$, Sigma) and DNase I $\left(100 \mathrm{mg} / \mathrm{ml}\right.$, Sigma) in DMEM with $10 \% \mathrm{FBS}$, at $37^{\circ} \mathrm{C}$ for 30 minutes, homogenized using gentle MACS (Miltenyi Biotech) and filtered through a $0.7 \mathrm{~mm}$ cell mesh. Cells were washed once with cold PBS and stored on ice. Flow cytometry analysis of cell surface and intracellular markers such as CD4, CD8, PD-1, OX40, ICOS, CTLA-4, Ki67 and Foxp3 etc. was performed as previously described ${ }^{47}$ using various antibodies as listed in the Supplementary Table 4. Briefly, cells were stained with the indicated mAbs in FACS buffer (0.5\%BSA in PBS, for all cell surface antigens) or permeabilization buffer (eBioscience, for Foxp3 intracellular antigens) for a minimum of 45 minutes on ice. All cytometric analysis was conducted on an LSR Fortessa instrument (BD Biosciences). Absolute cell numbers were quantified by flow cytometry using fluorescent reference beads (Thermo Fisher). Notably, intratumor $\mathrm{T}_{\text {reg }}$ cells expressed higher levels of CTLA-4 and there were no significant differences of $T_{\text {reg }}$ cells in spleen or lymph nodes from all the antibody treated groups, which was consistent with previous observations ${ }^{15}$.

\section{Quantification and statistical analysis}

Flow cytometry data were analyzed with FlowJo v10.4. Statistical analyses were done with Prism 7 (GraphPad Software). P values were calculated unpaired two-tailed Student's $t$ test or two-way ANOVA where indicated (ns, not significant; *, $p<0.05 ; \star \star, p<0.01 ; * \star \star, p<0.001 ; * \star \star \star, p<0.0001$ )

\section{References}

1. Brunet, J.-F. et al. A new member of the immunoglobulin superfamily-CTLA-4. Nature 328, 267 (1987).

2. Brunner, M. C. et al. CTLA-4-Mediated inhibition of early events of T cell proliferation. Journal of Immunology 162, 5813-5820 (1999).

3. Linsley, P. S. et al. CTLA-4 is a second receptor for the B cell activation antigen B7. Journal of Experimental Medicine 174, 561-569 (1991). 
4. Merwe, P. A. et al. CD80 (B7-1) binds both CD28 and CTLA-4 with a low affinity and very fast kinetics. Journal of Experimental Medicine 185, 393-403 (1997).

5. Oosterwegel, M. A. et al. CTLA-4 and T cell activation. Current Opinion in Immunology 11, 294-300 (1999).

6. Kajsa, W. et al. CTLA-4 control over Foxp3+ regulatory T cell function. Science 322, 271-275 (2008).

7. Leach, D. R. et al. Enhancement of antitumor immunity by CTLA-4 blockade. Science 271, 1734-1736 (1996).

8. Korman, A. J. et al. Checkpoint Blockade in Cancer Immunotherapy. Advances in Immunology 90, 297339 (2006).

9. Camacho, L. H. Novel therapies targeting the immune system: CTLA4 blockade with tremelimumab (CP-675,206), a fully human monoclonal antibody. Expert Opin Investig Drugs 17, 371-385 (2008).

10. Ramagopal, U. A. et al. Structural basis for cancer immunotherapy by the first-in-class checkpoint inhibitor ipilimumab. Proceedings of the National Academy of Sciences 114, E4223-E4232 (2017).

11. He, M. et al. Remarkably similar CTLA-4 binding properties of therapeutic ipilimumab and tremelimumab antibodies. Oncotarget 8, 67129-67139 (2017).

12. Lee, J. Y. et al. Structural basis of checkpoint blockade by monoclonal antibodies in cancer immunotherapy. Nature Communications 7, 13354-13354 (2016).

13. Ha, D. et al. Differential control of human Treg and effector T cells in tumor immunity by Fcengineered anti-CTLA-4 antibody. Proceedings of the National Academy of Sciences 116, 609-618 (2019).

14. Bulliard, Y. et al. Activating Fc $\mathrm{Y}$ receptors contribute to the antitumor activities of immunoregulatory receptor-targeting antibodies. Journal of Experimental Medicine 210, 1685-1693 (2013).

15. Simpson, T. R. et al. Fc-dependent depletion of tumor-infiltrating regulatory $T$ cells co-defines the efficacy of anti-CTLA-4 therapy against melanoma. Journal of Experimental Medicine 210, 1695-1710 (2013).

16. Selby, M. J. et al. Anti-CTLA-4 Antibodies of IgG2a Isotype Enhance Antitumor Activity through Reduction of Intratumoral Regulatory T Cells. Cancer Immunol Res 1, 32-42 (2013).

17. Vargas, F. A. et al. Fc effector function contributes to the activity of human anti-CTLA-4 antibodies. Cancer Cell 33, 649-663. e644 (2018).

18. Ingram, J. R. et al. Anti-CTLA-4 therapy requires an Fc domain for efficacy. Proceedings of the National Academy of Sciences of the United States of America 115, 3912-3917 (2018). 
19. Gao, H. et al. Structure of CTLA-4 complexed with a pH-sensitive cancer immunotherapeutic antibody. Cell Discovery 6, 79 (2020).

20. Du, X. et al. A reappraisal of CTLA-4 checkpoint blockade in cancer immunotherapy. Cell Res 28, 117 (2018).

21. Chao, Y. et al. Rigid-body ligand recognition drives cytotoxic T-lymphocyte antigen 4 (CTLA-4) receptor triggering. Journal of Biological Chemistry 286, 6685-6696 (2011).

22. Chuang, E. et al. Interaction of CTLA-4 with the Clathrin-Associated Protein AP50 Results in LigandIndependent Endocytosis That Limits Cell Surface Expression. Journal of Immunology 159, 144-151 (1997).

23. Qureshi, O. S. et al. Trans-endocytosis of CD80 and CD86: a molecular basis for the cell-extrinsic function of CTLA-4. Science 332, 600-603 (2011).

24. Najjar, Y. G. et al. Melanoma antigen-specific effector T cell cytokine secretion patterns in patients treated with ipilimumab. Journal of translational medicine 15, 39 (2017).

25. Clynes, R. A. et al. Inhibitory Fc receptors modulate in vivo cytoxicity against tumor targets. Nature Medicine 6, 443-446 (2000).

26. Stewart, R. et al. The role of Fc gamma receptors in the activity of immunomodulatory antibodies for cancer. Journal for Immunotherapy of Cancer 2, 29 (2014).

27. Romano, E. et al. Ipilimumab-dependent cell-mediated cytotoxicity of regulatory T cells ex vivo by nonclassical monocytes in melanoma patients. Proc Natl Acad Sci U S A 112, 6140-6145 (2015).

28. Sharma, A. et al. Anti-CTLA-4 immunotherapy does not deplete FOXP3+ regulatory T cells (Tregs) in human cancers. Clinical Cancer Research 25, 1233-1238 (2019).

29. Togashi, Y. et al. Regulatory T cells in cancer immunosuppression-implications for anticancer therapy. Nature Reviews Clinical Oncology, 1 (2019).

30. De Simone, M. et al. Transcriptional landscape of human tissue lymphocytes unveils uniqueness of tumor-infiltrating T regulatory cells. Immunity 45, 1135-1147 (2016).

31. Walker, L. S. et al. Confusing signals: recent progress in CTLA-4 biology. Trends in immunology 36, 63-70 (2015).

32. Linsley, P. S. et al. Intracellular Trafficking of CTLA-4 and Focal Localization Towards Sites of TCR Engagement. Immunity 4, 535-543 (1996).

33. Hye Sun, K. et al. Immune dysregulation in human subjects with heterozygous germline mutations in CTLA4. Science 345, 1623-1627 (2014). 
34. Desirée, S. et al. Autosomal dominant immune dysregulation syndrome in humans with CTLA4 mutations. Nature Medicine 20, 1410-1416 (2014).

35. Fecher, L. A. et al. Ipilimumab and its toxicities: a multidisciplinary approach. Oncologist 18, 733743 (2013).

36. Giacomo, A. M. D. et al. The Emerging Toxicity Profiles of Anti-CTLA-4 Antibodies Across Clinical Indications. Seminars in Oncology 37, 499-507 (2010).

37. Horvat, T. Z. et al. Immune-Related Adverse Events, Need for Systemic Immunosuppression, and Effects on Survival and Time to Treatment Failure in Patients With Melanoma Treated With Ipilimumab at Memorial Sloan Kettering Cancer Center. Journal of Clinical Oncology 33, 3193-3198 (2015).

38. Zhang, Y. et al. Hijacking antibody-induced CTLA-4 lysosomal degradation for safer and more effective cancer immunotherapy. Cell Research, 1 (2019).

39. Pardon, E. et al. A general protocol for the generation of Nanobodies for structural biology. Nature protocols 9,674 (2014).

40. L'Abbé, D. et al. Transient Gene Expression in Suspension HEK293-EBNA1 Cells: Methods and Protocols. (2018).

41. Collaborative, C. P. The CCP4 suite: programs for protein crystallography. Acta crystallographica. Section D, Biological crystallography 50, 760 (1994).

42. Storoni, L. C. et al. Likelihood-enhanced fast rotation functions. Acta Crystallographica Section D: Biological Crystallography 60, 432-438 (2004).

43. Emsley, P. et al. Coot: model-building tools for molecular graphics. Acta Crystallographica Section D: Biological Crystallography 60, 2126-2132 (2004).

44. Winn, M. et al. Use of TLS parameters to model anisotropic displacements in macromolecular refinement. Acta Crystallographica Section D: Biological Crystallography 57, 122-133 (2001).

45. Krissinel, E. et al. Inference of Macromolecular Assemblies from Crystalline State. Journal of Molecular Biology 372, 774-797 (2007).

46. Schrodinger, L. The PyMOL molecular graphics system. Version 1, 0 (2010).

47. Waight, J. D. et al. Cutting edge: epigenetic regulation of Foxp3 defines a stable population of CD4+ regulatory T cells in tumors from mice and humans. The Journal of Immunology 194, 878-882 (2015).

\section{Figures}


a

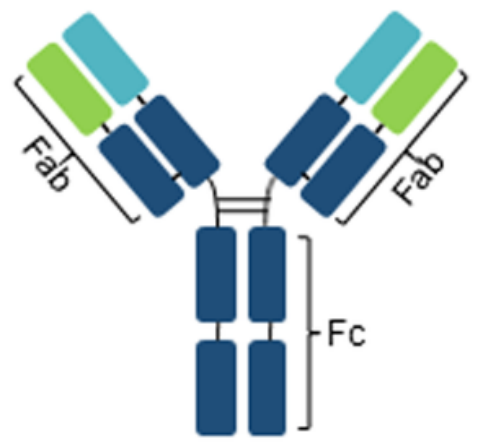

ipilimumab (Ipi)

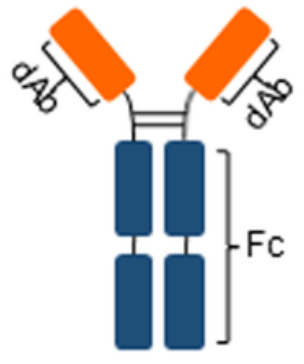

D138

b

D138

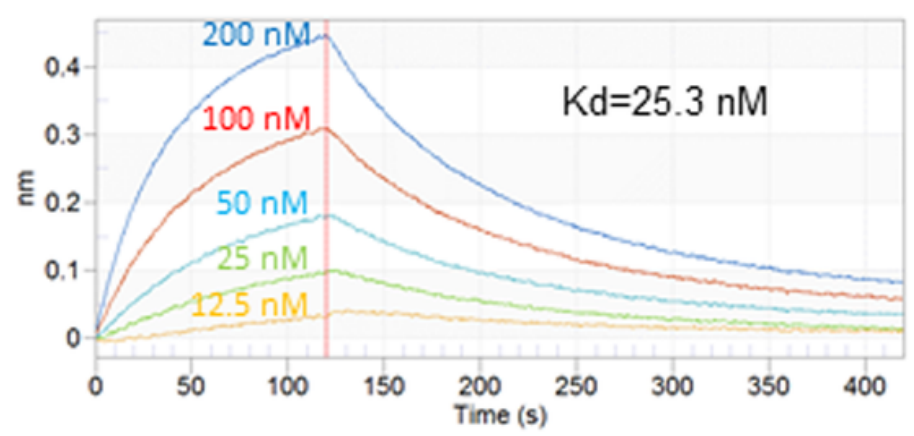

B7-2

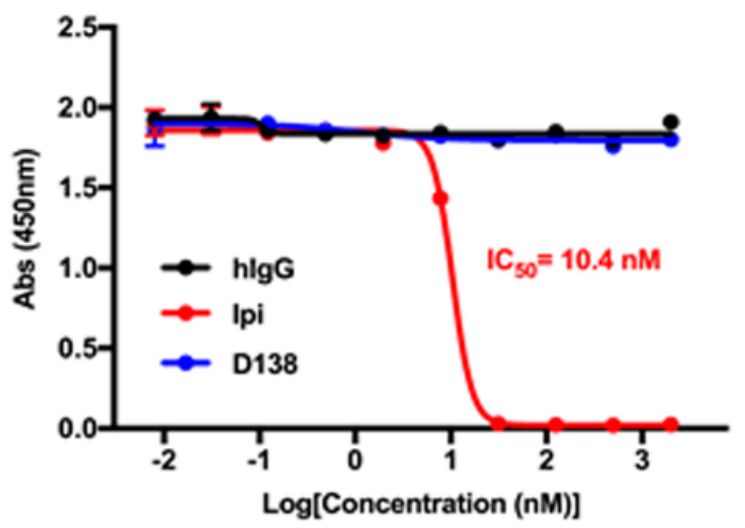

d

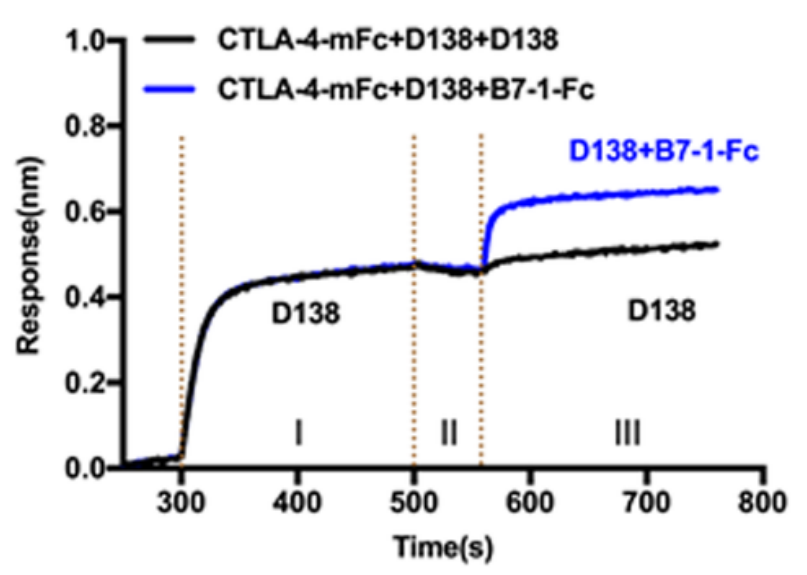

Figure 1

Characterization of the non-blocking antibody D138. a. Diagrams showing domains of ipilimumab and D138. b. D138 binds CTLA-4 with a Kd of $25.3 \mathrm{nM}$ when measured by BLI assay with D138 immobilized on the sensor. Different concentrations of CTLA-4-mFc (12.5-200 nM) was applied onto the sensor with the sensorgram recorded and fitted. c. ELISA assessment of the competitive binding of B7-1 (left) or B7-2 (right) at $50 \mathrm{ng} / \mathrm{ml}$ with ipilimumab (red) and D138 (blue) of different concentrations towards CTLA-4 coated on an ELISA plate. The bound B7 on plate was shown as the mean of optical density at $450 \mathrm{~nm}$ 
from triplicate wells $( \pm S D)$. d. BLI assessment of binding of B7-1 with preformed CTLA-4-D138 complex. The chips were first immobilized with CTLA-4-mFc, loaded with D138 (blue) for 200 seconds (stage I) and then washed for 60 seconds (stage II). Subsequently, sensor chips were loaded with 50 nM D138 alone (black) or D138 (50 nM) containing B7-1-Fc (200 nM) with the response signals recorded (stage III).

a

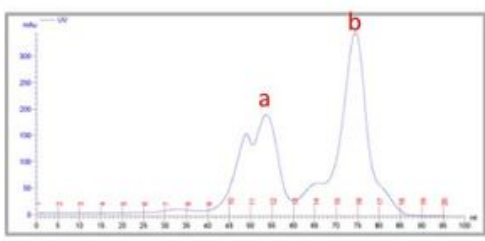

C

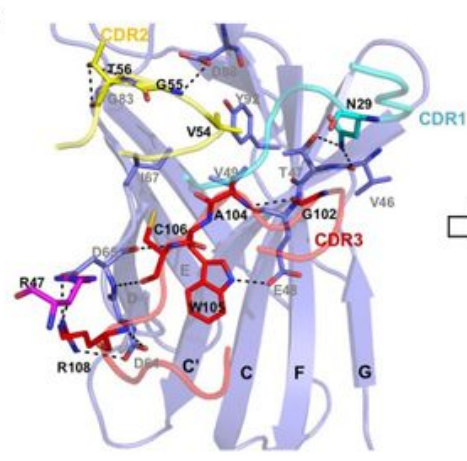

e

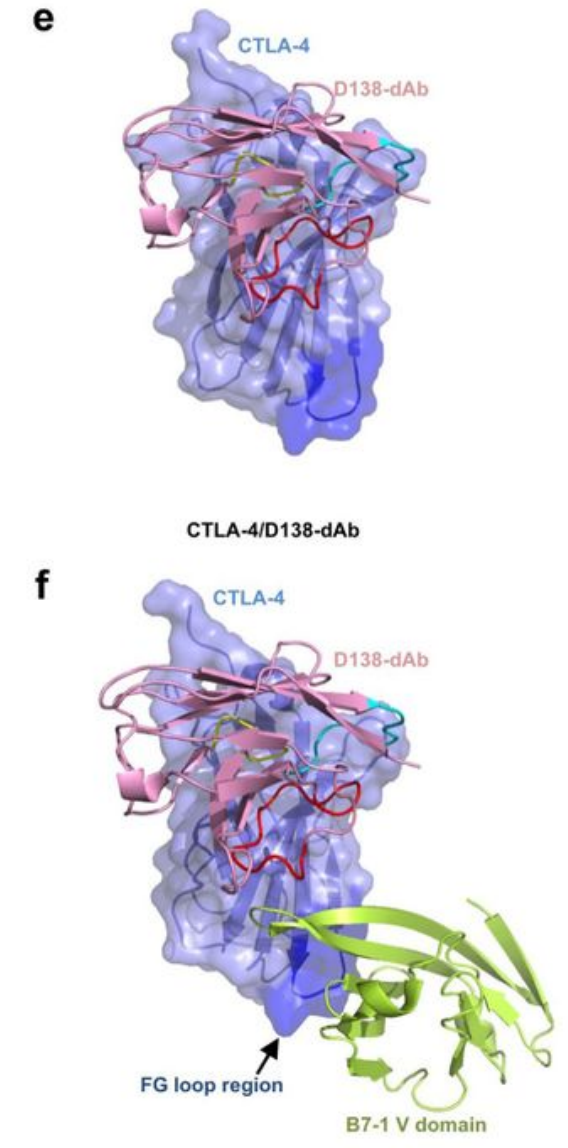

CTLA-4/D138-dAb

f

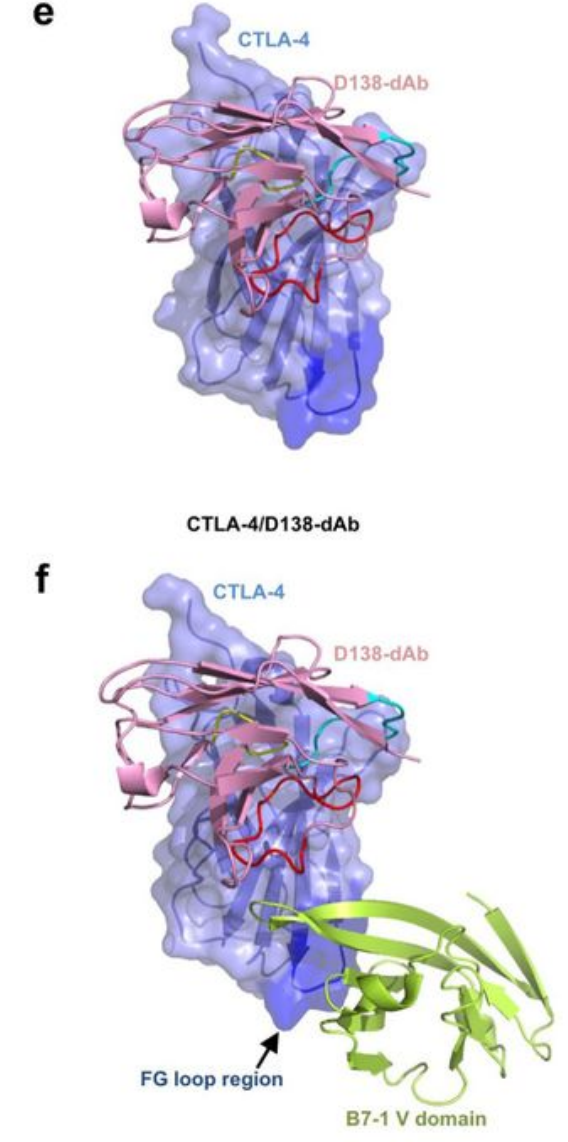

b

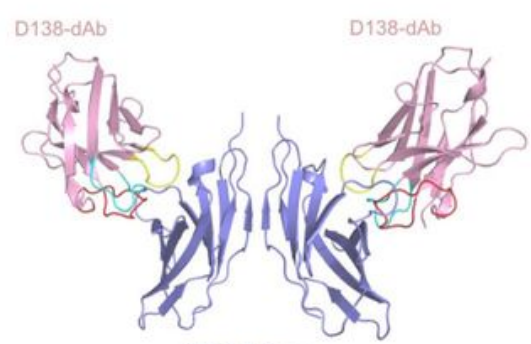

d

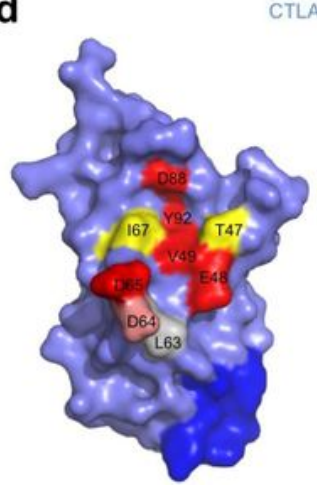

CTLA-4 dimer

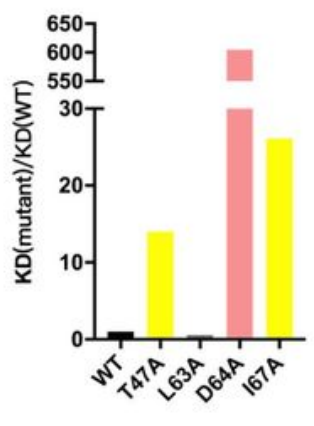

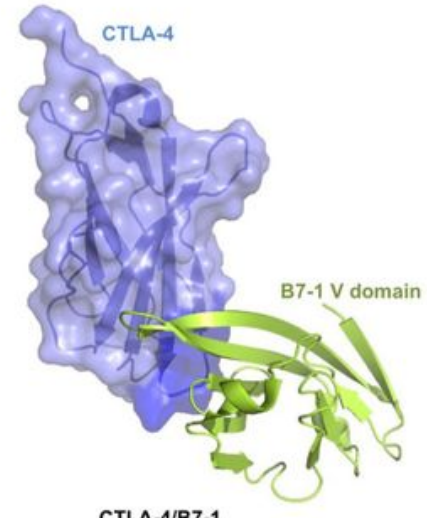

CTLA-4/B7-1

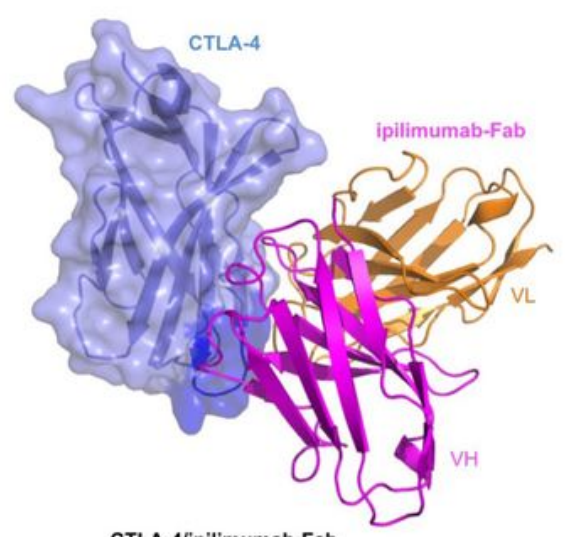

CTLA-4/ipilimumab-Fab

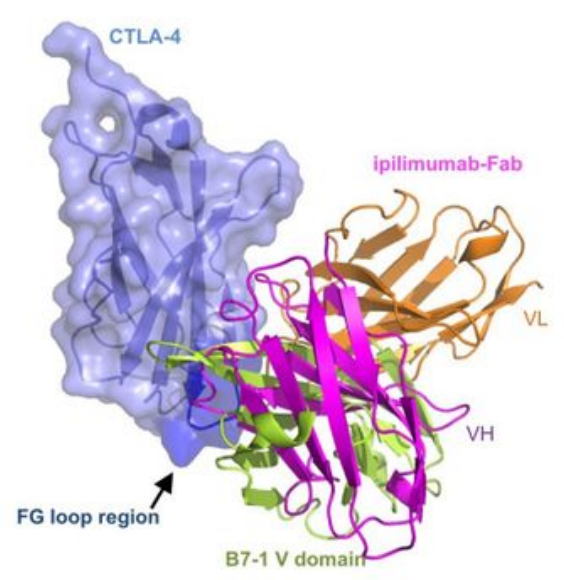

Figure 2 
Structural study of CTLA-4 complexed with D138 or ipilimumab. a. Preparation of CTLA-4 dimer complexed with D138-dAb. CTLA-4 dimer is formed by an intermolecular disulfide bond of Cys122. When complexed with D138-dAb, it was eluted in a later peak (b) on a SP ionic exchange column whereas CTLA-4 monomer/D138-dAb complex was eluted in an early peak (a) as visualized by SDS-PAGE analysis. b. The crystal structure of CTLA-4 dimer (blue) with two D138-dAb in a butterfly shape. c. Overall D138-dAb binds to a concave surface near the top of CTLA-4 involving both hydrophobic interactions and hydrogen bonding with key interactions shown. $d$. Mutagenesis analysis of CTLA-4 surface residues involved in complex formation showed replacement of E48, V49, D65, D88 and Y92 (colored in red) with Ala individually abolished the CTLA-4 binding towards D138 while replacement of T47, D64 and 167 also resulted significant decrease in binding affinity (Supplementary Table 3). e. The binding position of D138 on CTLA-4 is near the top of the molecule while both B7-1 and ipilimumab bind CTLA-4 on the lower part (blue) of the front beta-sheet. $\mathrm{f}$. Superimposing structures of CTLA-4-B7-1 complex with CTLA-4-D138 showed little clash between D138 and B7-1. In contrast, there are extensive clashes between B7-1 and ipilimumab when the corresponding structures were superposed together on CTLA-4. Only VH (purple) and VL (orange) domains of ipilimumab-Fab were shown for clarity. CTLA-4 is shown as semitransparent surface in slate, B7-1 is in green and D138 is in pink. 
a

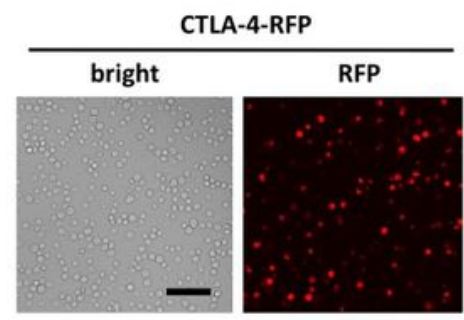

CTLA-4-RFP: B7-1-GFP=1: 1

b

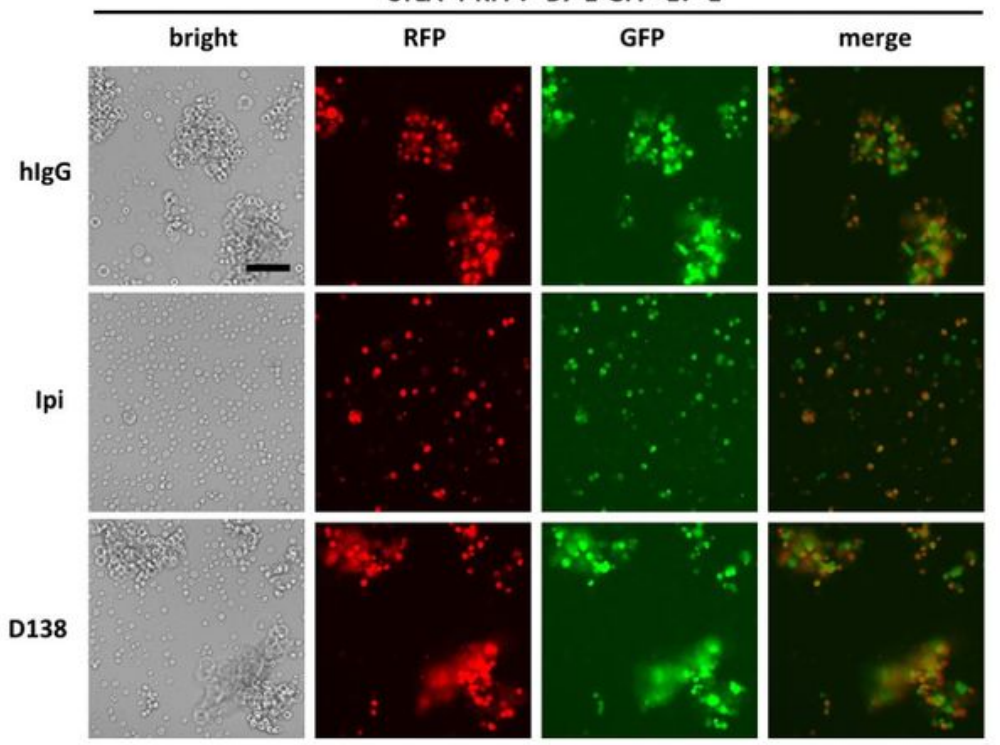

C

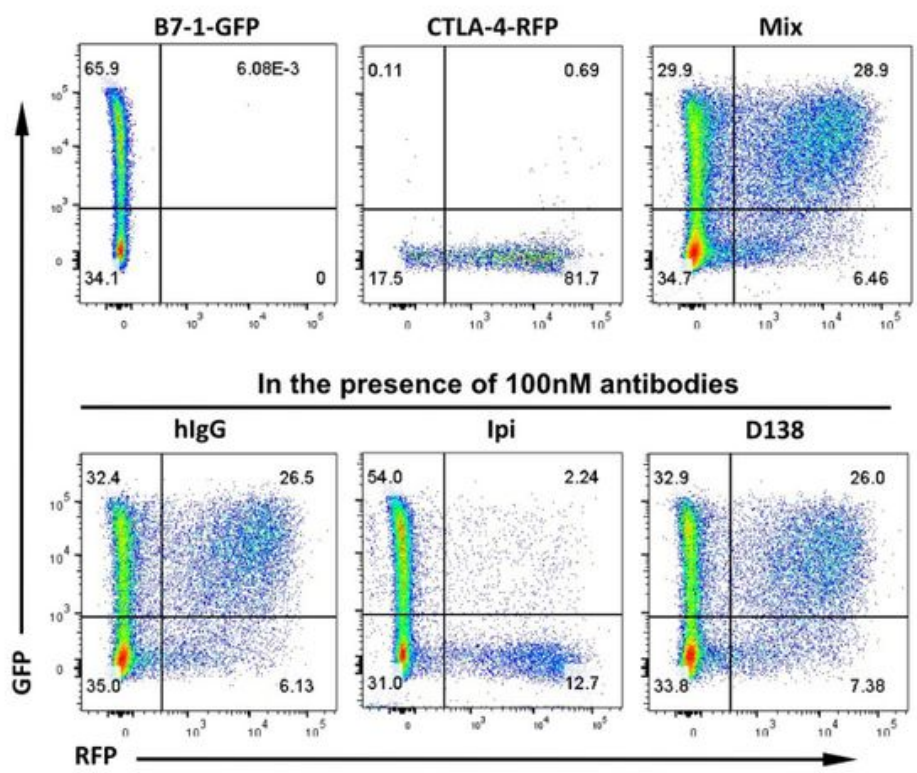

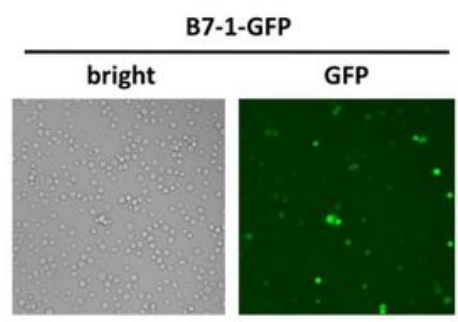

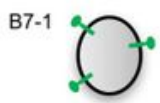

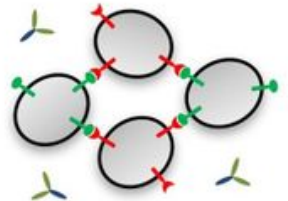
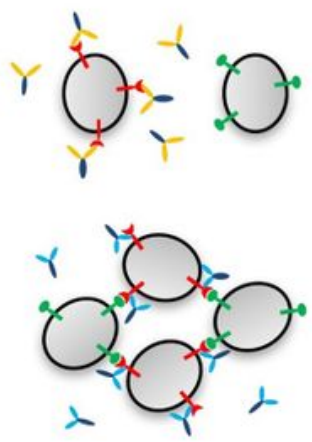

B7-1/CTLA-4
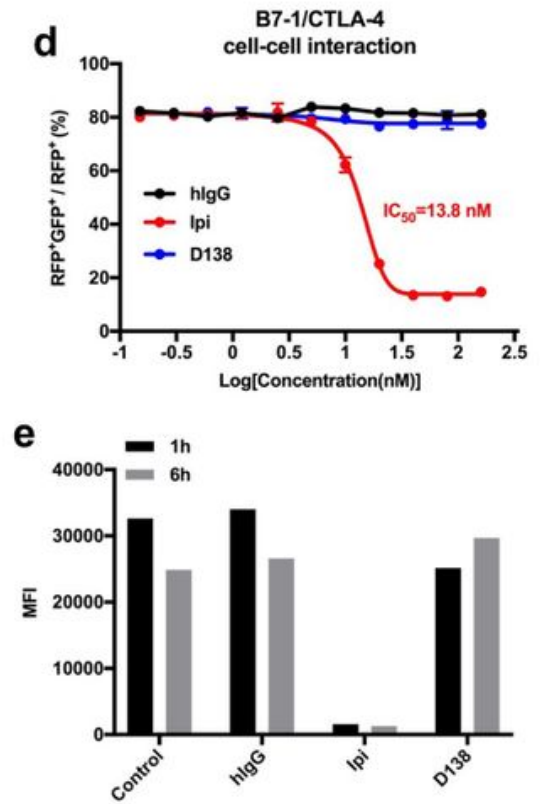

\section{Figure 3}

Effect of antibody on cell surface expressed CTLA-4 and B7-1. a. CTLA-4 mutant with its cytosolic tail replaced by that of PD-L1 to block endocytosis was fused with RFP and expressed in HEK293T cells showing red fluorescence. B7-1 was fused with GFP showing green florescence. b. Cells expressing CTLA4-RFP were treated with $100 \mathrm{nM}$ antibodies (hlgG, Ipi, D138) respectively and then mixed with cells expressing B7-1-GFP cells at $4^{\circ} \mathrm{C}$ for 5 minutes at 1:1 ratio before fluorescence microscope analysis. The 
cartoons illustrate the interactions of antibodies on cell surface. c. Flow cytometry analysis of cell-cell interactions. Cells expressing B7-1-GFP or CTLA-4-RFP or their mixture at 5:1 ratio were analyzed for both GFP and RFP signals (up panel). Cells expressing CTLA-4-RFP pretreated with 100nM antibodies were similarly mixed with B7-1-GFP cells and analyzed for the tethered cells (lower panel). $d$. The dose dependent blockage of cell-cell interaction by the antibodies. As in c, cells expressing CTLA-4-RFP were treated with concentrations of antibodies $(0.16 \mathrm{nM} 160 \mathrm{nM})$ and then mixed with B7-1-GFP cells before analysis by flow cytometry. Each sample was measured twice and the percentage of GFP+RFP+ double positive cells over RFP+ cells (Mean $\pm S D$ ) was plotted against the dose of the antibodies with EC50 fitted from the curve. e. Effects of antibody binding on the surface expressed CTLA-4. HEK293T cells with stably expressed human CTLA-4 were treated with $40 \mathrm{nM}$ of antibodies for 1 or $6 \mathrm{~h}$ at $37^{\circ} \mathrm{C}$, the cells were then washed and probed with a PE-labelled CTLA-4 antibody, which binds CTLA-4 on a different site to that of ipilimumab or D138, with cell surface fluorescence signal measured by flow cytometry. The assay was performed in triplicate wells with representative data shown.

a

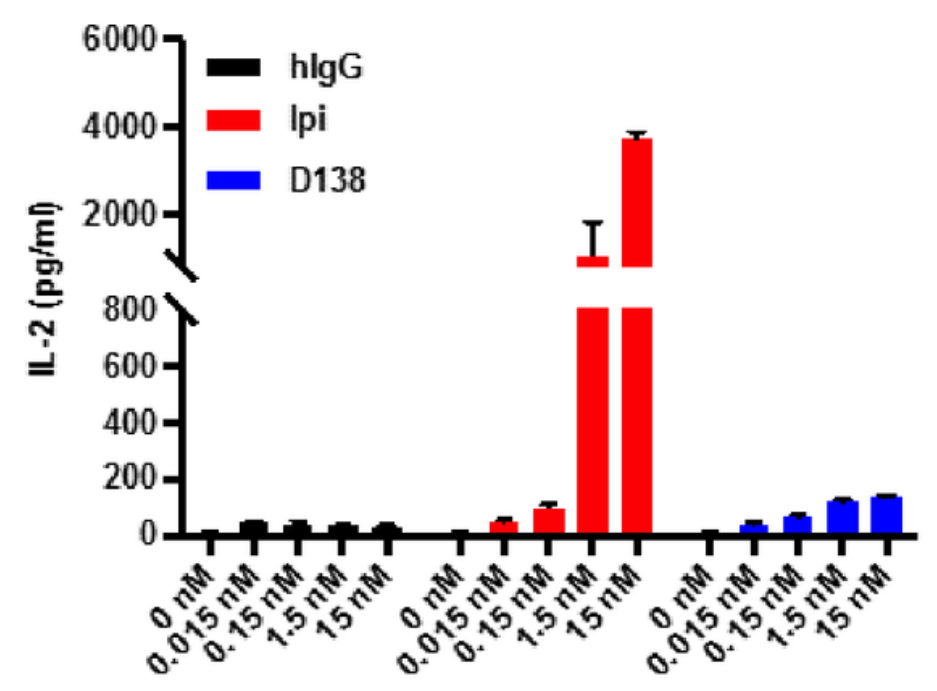

b

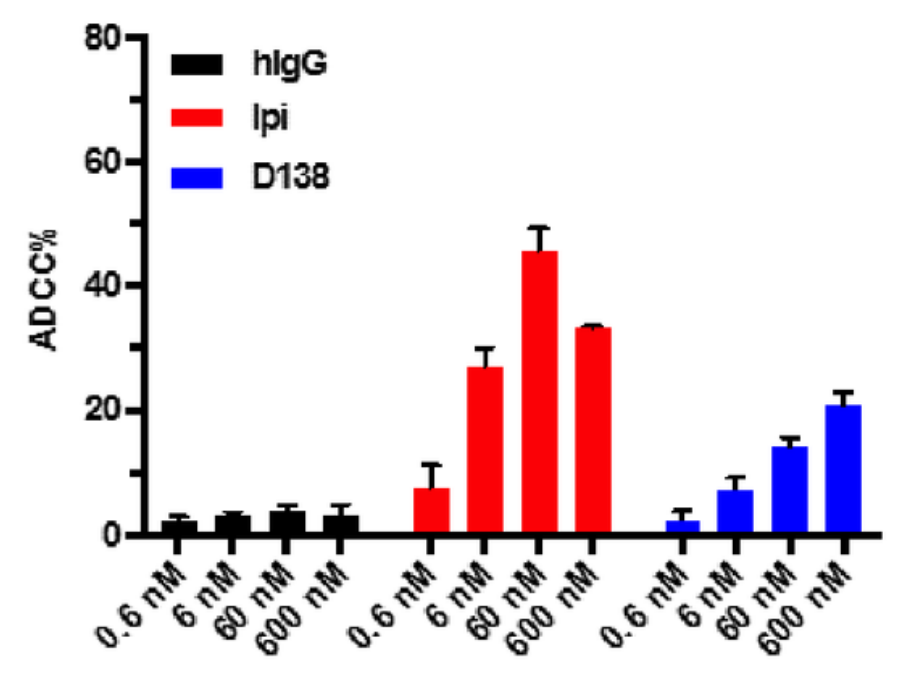

Figure 4

The biology activity of D138. a. IL-2 secretion induced by antibodies. PBMCs were treated with different concentrations of ipilimumab and D138. After stimulation, IL-2 secreted in the supernatant was measured after five days culture. Data represent mean $\pm S D(n=3)$. b. ADCC activity of the antibodies were determined using PBMC. Treg cells were then separated from the PBMCs, used as target cells. The PBMC were cultured and incubated with $30 \mathrm{ng} / \mathrm{ml} \mathrm{IL-2}$ for $22 \mathrm{~h}$ (effector: target=15:1), and along with different doses of CTLA-4 antibodies or control antibody. The effect of ADCC was measured by LDH release after 4 $h$ from treatments $(n=3)$. 

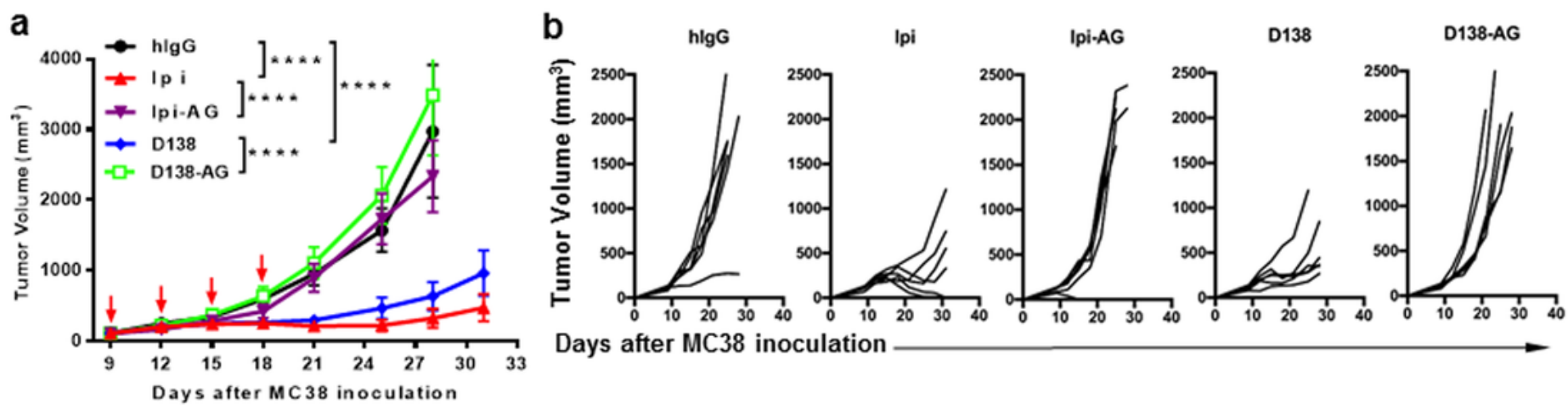

C
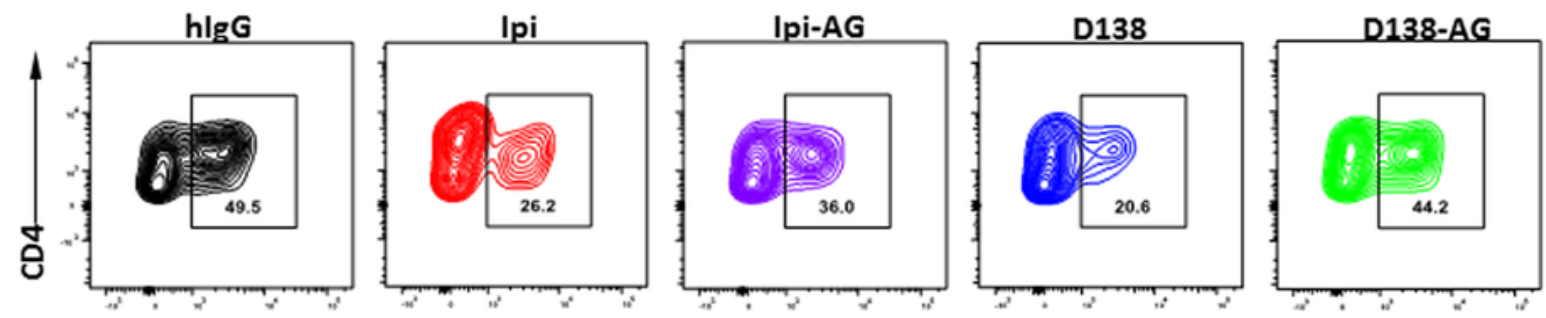

Foxp3
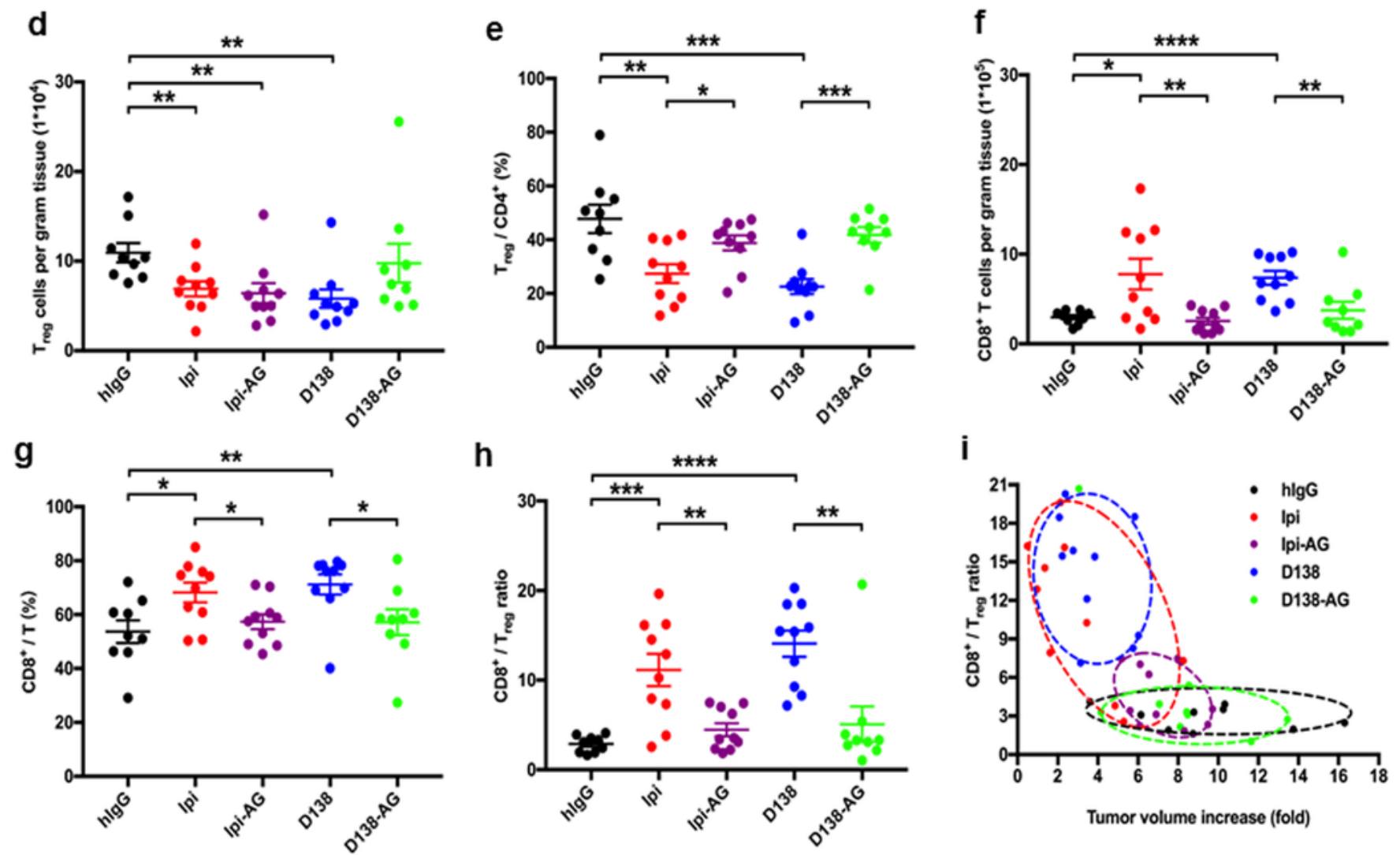

i

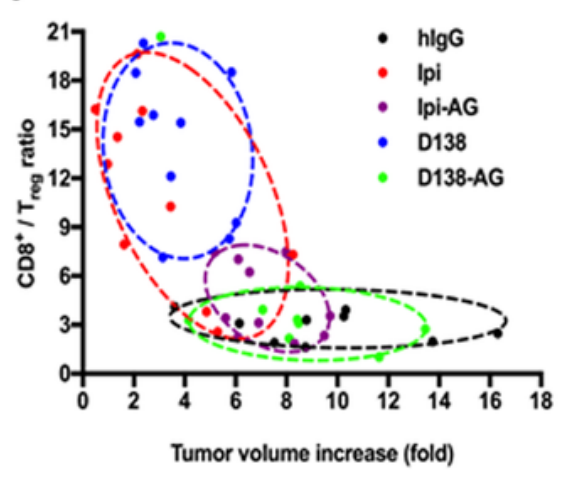

Figure 5

Antitumor effect of D138 and ipilimumab is Fc-dependent and associated with Treg depletion. a. Agematched Ctla4h/h C57BL/6J female mice were inoculated with MC38 (1'106 cells). After tumor size reached about $80 \sim 160 \mathrm{~mm} 3$, mice were grouped $(n=6)$ and treated (arrowed) via intraperitoneal administration of antibodies. The tumor sizes were measured with individual tumor growth shown in $b$. Data represent mean \pm S.E.M. and statistical analysis were performed by two-way repeated measures 
ANOVA. c. The contour plots representatively show the percentage of CD4+Foxp3+ $T$ cells subpopulation in CD4+ T cells. $d$-i. Intratumor T cell population analysis. Mice ( $n=10$ each group) were treated similarly as in $\mathrm{c}$ and sacrificed after four injections of antibodies (Supplementary Figure 2). Tumor cells were stained with various mAbs and analyzed with flow cytometry. e. Numbers of Treg cells per gram of tumor tissue. e. The percentage of Treg (CD4+Foxp3+) cells over total CD4+ T cells. f. Numbers of CD8+ cells per gram of tumor tissue. $\mathrm{g}$. The percentage of CD8+ T cells over total T cells. h. The ratio of CD8+ over Treg cells. i. The correlation between tumor growth with the ratio of CD8+/Treg cells in each tumor with each group circled together. Notably, one outlier in the D138-AG treated group was out.

\section{Supplementary Files}

This is a list of supplementary files associated with this preprint. Click to download.

- D1382021NCSupplementaryMaterials.docx 